\title{
Ultrasonographic Study of the Anatomical Relationship Between the Lateral Antebrachial Cutaneous Nerve and the Cephalic Vein
}

\author{
Hyung Soon Im, MD, Jin Young Im, MD, Ki Hoon Kim, MD, Dong Hwee Kim, MD, Byung Kyu Park, MD
}

Department of Physical Medicine and Rehabilitation, Korea University Ansan Hospital, Ansan, Korea

\begin{abstract}
Objective To define the anatomy of the lateral antebrachial cutaneous nerve (LABCN) and the cephalic vein (CV) in the anterior forearm region of living humans using ultrasonography for preventing LABCN injury during cephalic venipuncture.

Methods Thirty forearms of 15 healthy volunteers were evaluated using ultrasonography to identify the point where the LABCN begins to contact with the CV, and the point where the LABCN separates from the CV. The LABCN pathway in the forearm in relation to a nerve conduction study was also evaluated.

Results The LABCNs came in contact with the CV at a mean of $0.6 \pm 1.6 \mathrm{~cm}$ distal to the elbow crease, and separated from the $\mathrm{CV}$ at a mean of $7.0 \pm 3.4 \mathrm{~cm}$ distal to the elbow crease. The mean distance between the conventionally used recording points (point R) for the LABCN conduction study and the actual sonographic measured LABCN was $2.4 \pm 2.4 \mathrm{~mm}$. LABCN usually presented laterally at the point $\mathrm{R}(83.3 \%)$.

Conclusion The LABCN had close proximity to the CV in the proximal first quarter of the forearm. Cephalic venipuncture in this area should be avoided, and performed with caution if needed.
\end{abstract}

Keywords Lateral antebrachial cutaneous nerve, Phlebotomy, Ultrasonography

\section{INTRODUCTION}

The lateral antebrachial cutaneous nerve (LABCN) is a terminal branch of musculocutaneous nerve which is derived from the brachial plexus. It is responsible for sensing the lateral anterior aspect of the forearm [1]. The LABCN passes through the fascia of the biceps brachii muscle, and descends just lateral to the biceps brachii tendon around the elbow joint. There it finally pierces the deep fascia to become superficial. In some cases, the LABCN bifurcates into dorsal and volar branches. It also has an anatomical variation where the nerve innervates the radial border of the dorsum of the hand and thumb [2]. The cephalic vein (CV), also known as the antecubital

Received August 11, 2016; Accepted October 4, 2016

Corresponding author: Ki Hoon Kim

Department of Physical Medicine and Rehabilitation, Korea University Ansan Hospital, 123 Jeokgeum-ro, Danwon-gu, Ansan 15355, Korea. Tel: +8231-412-5330, Fax: +82-31-412-4215, E-mail: kkh702@korea.ac.kr

ORCID: Hyung Soon Im (http://orcid.org/0000-0003-0732-7690); Jin Young Im (http://orcid.org/0000-0003-0666-4580); Ki Hoon Kim (http://orcid. org/0000-0002-7257-3858); Dong Hwee Kim (http://orcid.org/0000-0002-8116-0078); Byung Kyu Park (http://orcid.org/0000-0001-8445-7099).

(c) This is an open-access article distributed under the terms of the Creative Commons Attribution Non-Commercial License (http://creativecommons.org/ licenses/by-nc/4.0) which permits unrestricted noncommercial use, distribution, and reproduction in any medium, provided the original work is properly cited. Copyright (c) 2017 by Korean Academy of Rehabilitation Medicine 
vein, is superficially located in the anterior aspect of elbow. It is one of the vessels commonly used for sampling blood. LABCN injuries can result from various conditions including direct nerve trauma, compressive neuropathies, neuritis, or due to neuromas and intraneural mucous cysts [3-5]. Because of the close proximity, injuring the LABCN during venipuncture of the CV has been reported in the several previous case reports [6-8]. Therefore, it is important to know the proper anatomy of these structures.

There have been several cadaver anatomical studies of the LABCN [9-11]. Among them, Wongkerdsook et al. [10] reported LABCN anatomy in relation to the lateral epicondyle and CV with a formalin-fixed cadaver. Also, Beldner et al. [11] reported that the LABCN ran parallel and volar to the cephalic vein in the forearm in $84 \%$ of specimens. However, it is difficult to say those cadaveric studies revealed an actual relationship between the LABCN and CV in a living human due to rigor mortis, cadaveric fixation, and desiccation, etc.

Currently, ultrasonographic evaluation is very useful for evaluating relationships in living human structures $[12,13]$. In this study, we obtain important information on the LABCN and CV using ultrasonography for safe venipuncture. For accurate nerve conduction study, we compared the positions of actual LABCNs to the conventionally used recording point for a nerve conduction study.

\section{MATERIALS AND METHODS}

We recruited healthy subjects who had no history of neuromuscular disease, were not taking any medication, and had no history of any trauma in the forearms or hands. We ultrasonographically studied 30 forearms of 15 volunteers (12 males and 3 females; mean age, $30.9 \pm 9.3$ years). Every subject participated in this study voluntarily with informed consent according to the Institutional Review Board of Korea University Ansan Hospital. We measured the height, weight, length and circumference of both forearms of each participant. The length of the forearm was defined as the distance from the medial epicondyle to the tip of ulnar styloid process. The forearm circumference was measured at the mid-point of the forearm length. A real time ultrasonography was performed using the
ACCUVIX V20 system (Samsung Medison, Seoul, Korea) interfaced with a 6-13 MHz linear array transducer. All procedures were conducted by a physiatrist experienced in musculoskeletal ultrasonography for consistent measurement throughout the study. The sonographer had over 8 years of experience using ultrasonography to visualize peripheral nerves, including cutaneous nerves. The transducer was always placed softly on the skin to prevent unnecessary movement of the soft tissue or collapse of the veins. We used built-in measurement tools for measuring the distance between the biceps tendon and the LABCN. A measuring tape was used for all measurements.

Ultrasonographic examinations of the LABCN and CV were performed in transverse view with subjects in the supine position, with their elbows extended and forearm supinated. First, a line was drawn in the elbow crease and the transducer was placed transversely to that line. The distance between the mid-point of each biceps tendon and the LABCN at the level of elbow crease was measured. Each biceps tendon and LABCN mid-point was defined as the crossing point of the shortest and longest diameters. Then, tracing the transducer distally along the path of the LABCN, we checked two points where the LABCN and CV meet (point M) and come apart (point A)

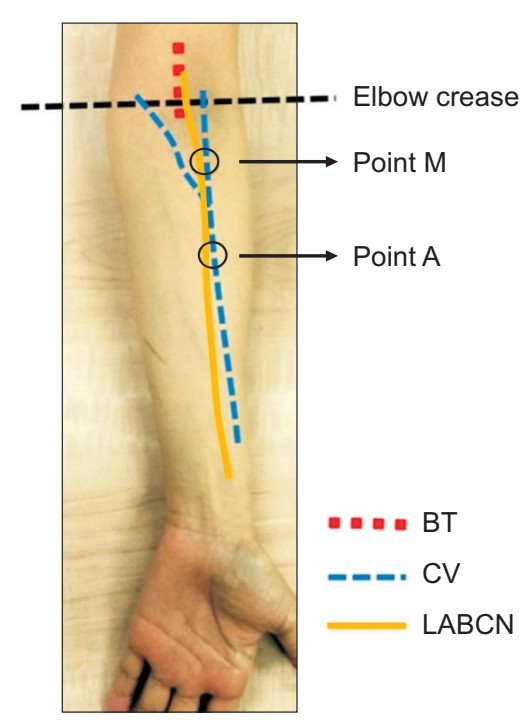

Fig. 1. Schematic relationship of the lateral antebrachial cutaneous nerve (LABCN), cephalic vein (CV), and biceps tendon (BT) at the anterior aspect of the forearm. Point M, meeting point of $\mathrm{LABCN}$ and CV; Point A, point where $\mathrm{LABCN}$ and $\mathrm{CV}$ come apart. 
(Fig. 1). Finally, we measured the distances between elbow crease and each point $\mathrm{M}$ and point $\mathrm{A}$, defining them as 'distance $\mathrm{M}^{\text {' }}$ and 'distance $\mathrm{A}$ '.

The LABCN recording point for the nerve conduction study using the antidromic method was named as 'point $\mathrm{R}$ ', and defined as a $12-\mathrm{cm}$ distal point from the elbow crease level on an imaginary line starting just lateral to the biceps tendon, and toward the radial artery at the wrist [14]. Using ultrasonography, we traced the LABCN from the elbow crease to the $12 \mathrm{~cm}$ distal to the elbow crease, and measured the distance of its actual location from the point R. We named this 'distance R'.

\section{RESULTS}

The mean height, weight, length of the forearm, and circumference of the forearm were $171.9 \pm 8.6 \mathrm{~cm}$, $65.0 \pm 9.8 \mathrm{~kg}, 24.8 \pm 2.0 \mathrm{~cm}$, and $22.1 \pm 1.7 \mathrm{~cm}$, respectively. The LABCN had a bright echogenic texture with hypoechoic fascicles (Fig. 2). The mean distance between the biceps brachii tendon and the LABCN in the level of elbow crease was $3.4 \pm 1.6 \mathrm{~mm}$. In all the cases, LABCNs were present laterally to the biceps tendon, and during its course, the LABCNs always met CV at least one time. Mean distance between the elbow crease and the point where the LABCN and CV meet (point M) was 0.6 41.6 $\mathrm{cm}$ distal to the elbow crease. Although in most of cases the LABCN and CV met below the elbow, in the 9 cases (30.0\%), they met above the elbow crease. The mean distance between the elbow crease and a point where the LABCN and CV come apart (point A) was $7.0 \pm 3.4 \mathrm{~cm}$ distal to the elbow crease. Finally, the LABCN investigated by sonography was located at a mean of $2.4 \pm 2.4 \mathrm{~mm}$ lateral to 'point $\mathrm{R}$ ' at $12 \mathrm{~cm}$ distal from the elbow crease (Table 1). Additionally, in only two forearms, LABCN bifurcation into volar and dorsal branches was observed via ultrasonography.

\section{DISCUSSION}

The present study evaluated the actual relationship between the LABCN and the CV in the cubital area by ul-
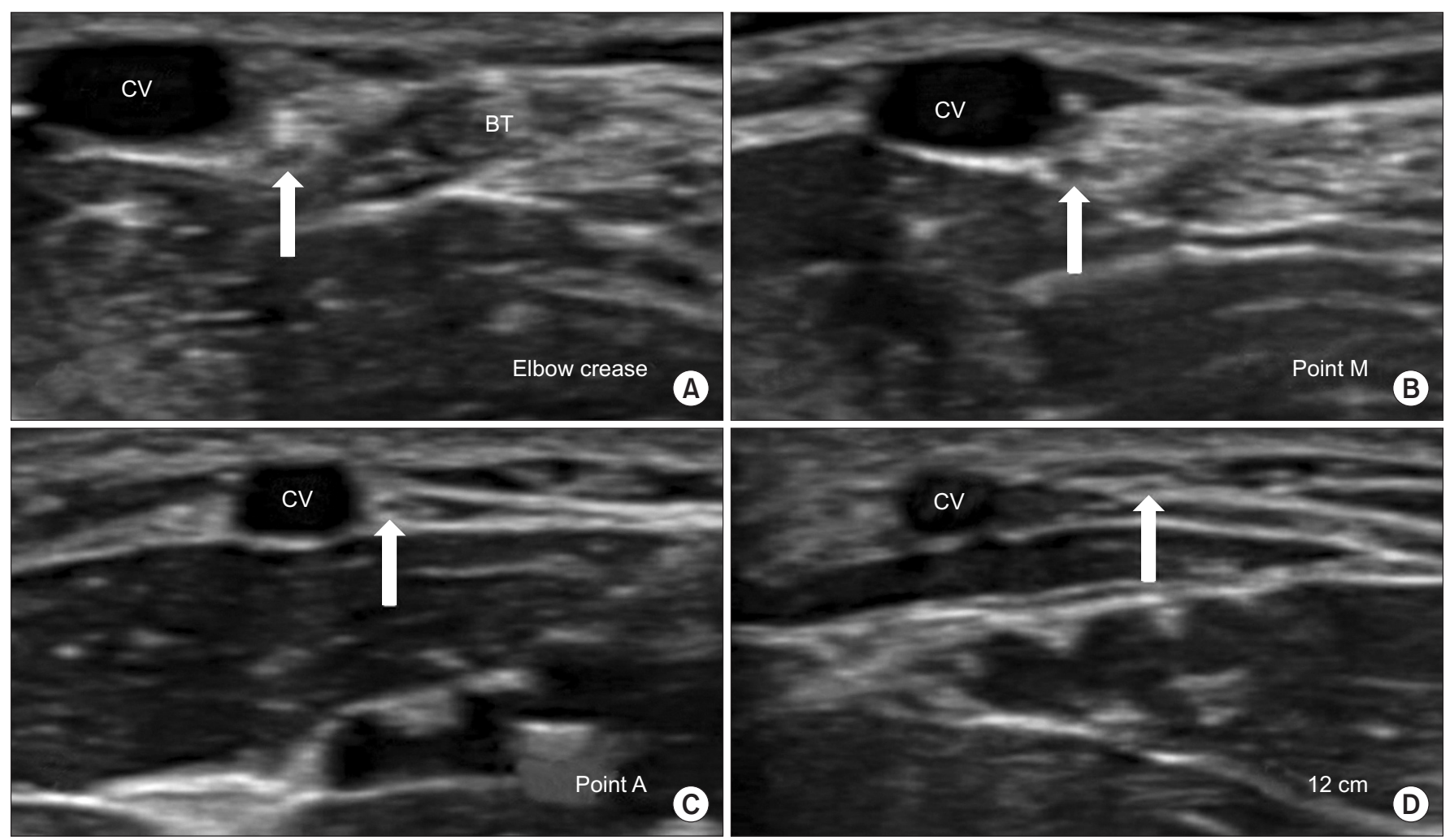

Fig. 2. Transverse view of ultrasonographic images of the course of the LABCN (arrows) at the elbow crease level (A), at the level of point $\mathrm{M}$ and $\mathrm{A}(\mathrm{B}, \mathrm{C})$, and at the distal $12 \mathrm{~cm}$ level (D). LABCN, lateral antebrachial cutaneous nerve; CV, cephalic vein; Point M, meeting point of LABCN and CV; Point A, point where LABCN and CV come apart. 
Table 1. The measured outcomes for evaluating the relationship of LABCN and CV

\begin{tabular}{lc}
\hline \multicolumn{1}{c}{ Parameter } & Value \\
\hline Forearm length $(\mathrm{cm})$ & $24.8 \pm 2.0(20.5$ to 27.5$)$ \\
Forearm circumference $(\mathrm{cm})$ & $22.1 \pm 1.7(19.0$ to 25.0$)$ \\
Distance of LABCN and BT $(\mathrm{mm})$ & $3.4 \pm 1.6(1.7$ to 7.7$)$ \\
Distance M $(\mathrm{cm})^{\mathrm{a})}$ & $-0.6 \pm 1.6(-3.7$ to 2.4$)$ \\
Distance A $(\mathrm{cm})^{\mathrm{a})}$ & $-7.0 \pm 3.4(-0.1$ to -13.5$)$ \\
Distance R $(\mathrm{mm})^{\mathrm{b})}$ & $2.4 \pm 2.4(-2.0$ to 7.5$)$ \\
\hline
\end{tabular}

Values are presented as mean \pm standard deviation (range). LABCN, lateral antebrachial cutaneous nerve; BT, distal tendon of the biceps brachii; Distance $\mathrm{M}$, distance between the elbow crease and the point where the LABCN and cephalic vein meet; Distance A, distance between the elbow crease and the point where the LABCN and cephalic vein come apart; Distance $\mathrm{R}$, distance between the LABCN and conventional recording point for nerve conduction study of the LABCN at the level $12 \mathrm{~cm}$ distal to the elbow crease.

${ }^{\text {a) }}$ Negative values represent distal position of meeting or separating point to the elbow crease.

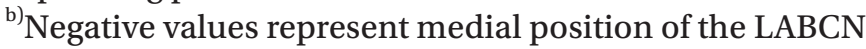
to the conventional recording point.

trasonography. There were some articles describing the course of the LABCN. McFarlane and Mayer reported a close relationship between the LABCN and CV in cadaveric dissections [9]. These veins and nerves were found consistently in all specimens midway between the skin and fascia of the forearm within the subcutaneous fat. The LABCN was found consistently at the same tissue depth as the cephalic vein. Wongkerdsook et al. [10] reported $89.6 \%$ of the LABCNs ran medial to the CV within $1 \mathrm{~cm}$ at the level of inter-epicondylar line and tended to course medial to the $\mathrm{CV}$ in the forearm.

In our study, there was a tendency that LABCN runs medially to the $\mathrm{CV}$ in the forearm as in the previous study. As we expected, there was very close proximity between the LABCN and CV around the cubital area, and they met at least once during its course. With our ultrasonographic data, the mean meeting point of the LABCN and CV is located distal to the elbow crease. Nevertheless, in the $30.0 \%$ of the cases (9 cases in our study), the LABCNs adhered to the $\mathrm{CV}$ at the elbow crease level such that they can be damaged by any kind of medical approach to the CV (such as sampling blood or inserting catheter, etc.). Injury to the LABCN can lead to various sensory symptoms like numbness or a burning sensation. Although venipuncture related nerve injuries occur infrequently, electromyographers and other related clinicians should be aware of this uncommon but clinically and medico-legally important phenomenon. If a patient complains about symptoms after procedures targeted to the $\mathrm{CV}$, clinicians have to consider the possibility of damage to the LABCN. Rayegani and Azadi [8] recommended electrodiagnostic study in patients complaining of neurologic symptoms at least 10 days after venipuncture. Unrecognized injury to this nerve, can lead to neuromas that may be difficult to treat. Therefore, proximal approaching for the $\mathrm{CV}$ above the elbow crease level during a medical procedure can be a safe option.

Although the distribution of the LABCN has been described in many above-mentioned articles, the majority of the studies have focused on the LABCN of cadavers. Therefore, to our knowledge, this is the first attempt to evaluate the anatomic relationship between the LABCN and the $\mathrm{CV}$ in living subjects through ultrasonographic examination.

As a limitation of this study, inter-observer variability of the ultrasonography was not analyzed. A small cutaneous nerve in the forearm may not be found according to the experience of the examiner in peripheral nerve ultrasonography. In this study, if the LABCN of the mid-forearm was not detected on the first attempt, it was reevaluated. We found LABCN bifurcation to volar and dorsal branches in only two forearms possibly due to the dorsal branch being too small to be seen by ultrasonography. Further study on the anatomical variation of the LABCN branches should be considered.

In conclusion, we identified a very close proximity between the LABCN and CV around the cubital area through ultrasonography. The LABCN had close approximation to the $\mathrm{CV}$ in the proximal first quarter of the forearm. This means that cephalic venipuncture in the cubital area requires careful attention to the possibility of nerve damage. We recommend cephalic venipuncture to be carried out in a more proximal part of the cubital area based on the results of this study. Also, a better value for studying LABCN nerve conduction can be achieved by positioning the recording electrode about 2 to $3 \mathrm{~mm}$ lateral to the conventionally used recording point. 


\section{CONFLICT OF INTEREST}

No potential conflict of interest relevant to this article was reported.

\section{REFERENCES}

1. Olson IA. The origin of the lateral cutaneous nerve of forearm and its anaesthesia for modified brachial plexus block. J Anat 1969;105(Pt 2):381-2.

2. Davidovich ER, Nascimento OJ. Superficial radial nerve-lateral antebrachial cutaneous nerve anatomic variation. Brain Behav 2014;4:70-4.

3. Bassett FH 3rd, Nunley JA. Compression of the musculocutaneous nerve at the elbow. J Bone Joint Surg Am 1982;64:1050-2.

4. Belzile E, Cloutier D. Entrapment of the lateral antebrachial cutaneous nerve exiting through the forearm fascia. J Hand Surg Am 2001;26:64-7.

5. Brogan DM, Bishop AT, Spinner RJ, Shin AY. Lateral antebrachial cutaneous neuropathy following the long head of the biceps rupture. J Hand Surg Am 2012;37: 673-6.

6. Sander HW, Conigliari MF, Masdeu JC. Antecubital phlebotomy complicated by lateral antebrachial cutaneous neuropathy. N Engl J Med 1998;339:2024.

7. Stevens RJ, Mahadevan V, Moss AL. Injury to the lateral cutaneous nerve of forearm after venous cannulation: a case report and literature review. Clin Anat
2012;25:659-62.

8. Rayegani SM, Azadi A. Lateral antebrachial cutaneous nerve injury induced by phlebotomy. J Brachial Plex Peripher Nerve Inj 2007;2:6.

9. McFarlane RM, Mayer JR. Digital nerve grafts with the lateral antebrachial cutaneous nerve. J Hand Surg Am 1976;1:169-73.

10. Wongkerdsook W, Agthong S, Amarase C, Yotnuengnit P, Huanmanop T, Chentanez V. Anatomy of the lateral antebrachial cutaneous nerve in relation to the lateral epicondyle and cephalic vein. Clin Anat 2011;24:5661.

11. Beldner S, Zlotolow DA, Melone CP Jr, Agnes AM, Jones $\mathrm{MH}$. Anatomy of the lateral antebrachial cutaneous and superficial radial nerves in the forearm: a cadaveric and clinical study. J Hand Surg Am 2005;30:1226-30.

12. Padua L, Hobson-Webb LD. Ultrasound as the first choice for peripheral nerve imaging? Neurology 2013; 80:1626-7.

13. Zaidman CM, Seelig MJ, Baker JC, Mackinnon SE, Pestronk A. Detection of peripheral nerve pathology: comparison of ultrasound and MRI. Neurology 2013; 80:1634-40.

14. Lee HJ, DeLisa JA. Manual of nerve conduction study and surface anatomy for needle electromyography. 4th ed. Philadelphia: Lippincott Williams \& Wilkins; 2005. p. 59. 